\title{
BMJ Open Optimised anthropometric indices as predictive screening tools for metabolic syndrome in adults: a cross- sectional study
}

Yating Wu, ${ }^{1}$ Hongyu Li, ${ }^{2}$ Xiujuan Tao, ${ }^{1}$ Yanna Fan, ${ }^{1}$ Qinghan Gao, ${ }^{1}$ Jianjun Yang (D) ${ }^{1}$

To cite: Wu Y, Li H, Tao X, et al. Optimised anthropometric indices as predictive screening tools for metabolic syndrome in adults: a crosssectional study. BMJ Open 2021;11:e043952. doi:10.1136/ bmjopen-2020-043952

- Prepublication history and additional material for this paper is available online. To view these files, please visit the journal online (http://dx.doi.org/10. 1136/bmjopen-2020-043952)

Received 18 August 2020 Revised 15 December 2020 Accepted 13 January 2021
Check for updates

(c) Author(s) (or their employer(s)) 2021. Re-use permitted under CC BY-NC. No commercial re-use. See rights and permissions. Published by BMJ.

${ }^{1}$ School of Public Health and Management, Ningxia Medical University, Yinchuan, China

${ }^{2}$ Public Health Division, Yinchuan City Center for Disease Control and Prevention, Yinchuan, China

Correspondence to

Professor Jianjun Yang; yangjianjun_1969@163.com

\section{ABSTRACT}

Objectives We aimed to evaluate the association between metabolic syndrome (MetS) and two latest optimised indices, waist divided by height ${ }^{0.5}$ (WHT.5R) and body mass index (BMI) multiply by the square root of $\mathrm{BMI}$ and waist circumference (BMI $\sqrt{ } \mathrm{WC})$, and to comprehensively compare the ability of 10 anthropometric indices to identify MetS, including BMI $\sqrt{ }$ WC, WHT.5R, abdominal volume index (AVI), waist-to-height ratio (WHtR), Body Roundness Index (BRI).

Design Cross-sectional study.

Setting Ningxia, China.

Participants A total of 3860 adults aged 18-88 years from Ningxia, China were recruited by a single stratified cluster random sampling method.

Methods ORs and 95\% Cls for associations between indices and MetS were calculated using binary logistic regression, and areas under the receiver operating characteristic (ROC) curves (AUCs) were performed to compare their predictive capacity for MetS. All results for men and women were analysed and presented separately. Results The highest adjusted ORs for MetS were observed for the following indices: AVI (OR 15.22, 95\% Cl 10.88 to 21.30 ), WHT.5R (OR 13.83, $95 \% \mathrm{Cl} 9.89$ to 19.35) and $\mathrm{BMI} / \mathrm{WC}(\mathrm{OR} 12.14,95 \% \mathrm{Cl} 8.79$ to 16.76$)$ in men, whereas BRI, WHtR (both OR 14.91, 95\% Cl 10.39 to 21.4), WHT.5R (OR $14.22,95 \% \mathrm{Cl} 9.96$ to 20.29 ) and AVI (OR 14.03, 95\% Cl 9.91 to 19.87) in women. In ROC analysis, AVI (AUC: 0.767 for men and 0.749 for women) and WHT.5R (AUC: 0.761 for men and 0.747 for women) showed the highest predictive ability for MetS.

Conclusions BMI $\sqrt{ }$ WC and WHT.5R were significantly positively associated with MetS, and AVI and WHT.5R could be useful screening tools for identifying MetS in both sexes.

\section{INTRODUCTION}

Metabolic syndrome (MetS) is known as a cluster of risk factors for cardiovascular disease and type 2 diabetes mellitus (T2DM), ${ }^{1}$ and is associated with kidney disease ${ }^{2}$ and many cancers. ${ }^{3}$ A review article has reported that obesity is fundamental to MetS as it appears to precede the emergence of the other MetS risk factors. ${ }^{4}$ Obesity and MetS have become global major health concern. Despite MRI
Strengths and limitations of this study

This is the first study that reports the association between waist divided by height ${ }^{0.5}$, body mass index $\sqrt{\text { waist circumference and metabolic syndrome }}$ (MetS), and that comprehensively compares the ability of 10 anthropometric indices in identifing MetS.

- The present study performed sensitivity analysis and found that the results did not change significantly.

- This is a cross-sectional design study, with its inherent limited explanation of cause-and-effect temporality.

- The present study defined MetS using International Diabetes Federation 2009 criteria, and therefore, further studies are needed to determine whether the results are consistent under different criteria.

and CT are considered 'gold standards' for the measurement of fat distribution, the cost and time of the imaging methods required is prohibitive in many settings. ${ }^{5}$ Fortunately, simple, non-invasive and inexpensive anthropometric measurements can evaluate excess adiposity. From a public health perspective, it is therefore important to identify simple and precise anthropometric measurements that reflect adipose tissue distribution and that closely relate to obesity and MetS.

Body mass index (BMI) is the most commonly recommended and used indice to categorise general obesity in clinical and epidemiological studies. However, BMI cannot distinguish muscle mass from fat mass, or reflect fat distribution, and is sometimes affected by age and gender. ${ }^{6}$ Alternatively, abdominal obesity indices, such as waist circumference (WC), waist-to-hip ratio (WHR), waist-to-height ratio (WHtR), Body Adiposity Index (BAI), conicity index (C-Index) and abdominal volume index (AVI), have been proposed, ${ }^{7-10}$ because they modulate the limitation of BMI. AVI 
was proposed as a reliable tool for estimation of overall abdominal volume that was shown to be strongly related to T2DM. ${ }^{10}$ Moreover, Body Roundness Index (BRI) and A Body Shape Index (ABSI) were proposed for that they can estimate body fat (BF) distributions. ${ }^{11} 12$ BRI was reported to be a predictor of $\mathrm{BF}$ and visceral adipose tissue, ${ }^{11}$ and was associated with MetS and insulin resistance. ${ }^{13} \mathrm{ABSI}$ was found to be superior to BMI as predictor of mortality hazard. ${ }^{12}$ Recently, two new anthropometric indices have been introduced. ${ }^{14}{ }^{15}$ In 2016, Nevill et al ${ }^{14}$ developed 'waist divided by height ${ }^{0.5}$, (WHT.5R), and demonstrated that WHT.5R was a waist girth index that was independent of stature, and a stronger predictor of cardiometabolic risk than other anthropometric indices such as WHtR. A recent analytical review provided further support for WHT.5R, which has the strongest association with adiposity while having the weakest correlation with height. ${ }^{16}$ In 2019, another new index, BMI multiply by the square root of WC (BMI $\sqrt{W C}$ ), was proposed by using allometric modelling approach in four independent samples, and provided encouraging evidence that $\mathrm{BMI} \sqrt{ } \mathrm{WC}$ was effective in evaluating percentage $\mathrm{BF}$ in children. ${ }^{15}$

There have been many epidemiology investigations on the association between anthropometric indices and MetS, however, the aforementioned study did not examine the association between WHT.5R, BMI $\sqrt{W C}$ and MetS, and the anthropometric indices compared by previous studies were not identical and comprehensive. Moreover, it is still controversial which the best indice for identifying MetS is. Thus, this study aims, first, to estimate the association between MetS and two latest indices, WHT.5R and BMI $\sqrt{W C}$, second, to comprehensively compare the ability of BMI, BMI $\sqrt{ } \mathrm{WC}, \mathrm{WHR}, \mathrm{WHtR}, \mathrm{WHT} .5 \mathrm{R}$, BRI, BAI, ABSI, C-Index, AVI in identifying MetS from a group of adults in northwest China.

\section{METHODS}

\section{Study population}

This was a cross-sectional study, conducted in July 2010 to November 2011. A total of 4718 subjects were selected using single stratified cluster random sampling in Yinchuan, Wuzhong, Zhongwei, Guyuan and Shizuishan in Ningxia, which is located in northwest China. In the present study, we excluded participants who were under 18 years old or who were pregnant or had cachexia; participants with missing anthropometric, or laboratory measurements were excluded. Visual inspection of the data using boxplots revealed 95 outliers, the population height $<1.4 \mathrm{~m}$, body weight $<35 \mathrm{~kg}$, BMI $>50 \mathrm{~kg} / \mathrm{m}^{2}$, WC $>110.5 \mathrm{~cm}$ and HDL $>3.5 \mathrm{mmol} / \mathrm{L}$. Data were finally analysed from 3860 participants (aged 18-88 years), each with complete anthropometric measurements, biochemical indicator and covariable data (figure 1).

\section{Patient and public involvement statement}

No patient and public involvement was undertaken.

\section{Anthropometric measurements}

Anthropometric measurements were collected by trained staff following standard procedures. Subjects wore light indoor clothing without shoes during body weight and height measurements. WC was measured over the midpoint between the lower border of the ribs and iliac crest in the midaxillary plane. Hip circumference (HC) was measured at the maximum protuberance of the buttocks, which coincides in the front with the symphysis pubis.

\section{Calculation of anthropometric indices}

BMI, BMI $\sqrt{ }$ WC, WHR, WHtR, WHT.5R, BRI, BAI, ABSI, C-Index, AVI were calculated using the following formulas.

- BMI=weight $(\mathrm{kg}) /$ height $(\mathrm{m})^{2}$.

- BMI $\sqrt{W C}=B M I \times \sqrt{\mathrm{WC}(\mathrm{m})^{15}}$.

- $\mathrm{WHR}=\mathrm{WC}(\mathrm{cm}) / \mathrm{HC}(\mathrm{cm})$.

- WHtR=WC $(\mathrm{cm}) /$ height $(\mathrm{cm})$.

- WHT.5R=WC $(\mathrm{cm}) /$ height $^{0.5}(\mathrm{~cm})^{16}$.

- BRI=364.2-365.5 [1 - ळ-2 $\mathrm{WC}^{2}(\mathrm{~m})$ Height $\left.-2(\mathrm{~m})\right]$ $1 / 211$

- BAI=HC $(\mathrm{m}) /$ Height $^{1.5}(\mathrm{~m})-18^{8}$.

- ABSI $=W C(\mathrm{~m}) /\left[\mathrm{BMI}^{2 / 3}\left(\mathrm{~kg} / \mathrm{m}^{2}\right) \text { Height }^{1 / 2}(\mathrm{~m})\right]^{12}$.

- C-Index $=\mathrm{WC}(\mathrm{m}) /[0.109 \times \sqrt{\text { weight }(\mathrm{kg}) / \text { height }(\mathrm{m})}$ ]$^{9}$.

- AVI $=\left[2 \mathrm{~cm}(\mathrm{WC})^{2}+0.7 \mathrm{~cm}(\mathrm{WC}-\mathrm{HC})^{2} / 1000^{10}\right.$.

\section{Cardiometabolic risk parameters measurements}

Systolic and diastolic blood pressure (SBP and DBP, mm $\mathrm{Hg}$ ) were determined in triplicate with use of validated electronic manometer after 5 mins of rest in a sitting position, and the average of three repeated measurements was taken into analysis. Blood samples collected after overnight fast were used to determine serum fasting glucose (FBG, mmol/L), total cholesterol (TC, $\mathrm{mmol} / \mathrm{L}$ ), high-density lipoprotein cholesterol (HDL-C, mmol/L), low-density lipoprotein cholesterol (LDL-C, mmol/L) and triglyceride (TG, mmol/L) levels by standard laboratory enzymatic methods (Hitachi 7060, Tokyo, Japan). Non-HDL cholesterol (Non-HDL-C) was calculated as TC minus HDL-C. ${ }^{17}$

\section{Diagnostic criteria of MetS}

MetS was defined according to the definition of the International Diabetes Federation (IDF) criteria with Chinesespecific abdominal obesity standard. ${ }^{1}$ MetS needs at least three of following risk factors: (1) abdominal obesity (WC: men $\geq 85 \mathrm{~cm}$, women $\geq 80 \mathrm{~cm}$ ); (2) elevated TG (TG $\geq 1.7 \mathrm{mmol} / \mathrm{L}$ ); (3) low HDL-C (HDL-C: men $<1.0 \mathrm{mmol} / \mathrm{L}$ ), women $<1.3 \mathrm{mmol} / \mathrm{L}$ ); (4) elevated BP (SBP $\geq 130 \mathrm{~mm} \mathrm{Hg}$ or DBP $\geq 85 \mathrm{~mm} \mathrm{Hg}$, or using antihypertension drugs) and (5) elevated (FBG $\geq 5.6 \mathrm{mmol} / \mathrm{L}$ or patients previously diagnosed with diabetes).

\section{Covariables assessment}

Data on demographic characteristics, lifestyle habits and personal medical information were collected with 


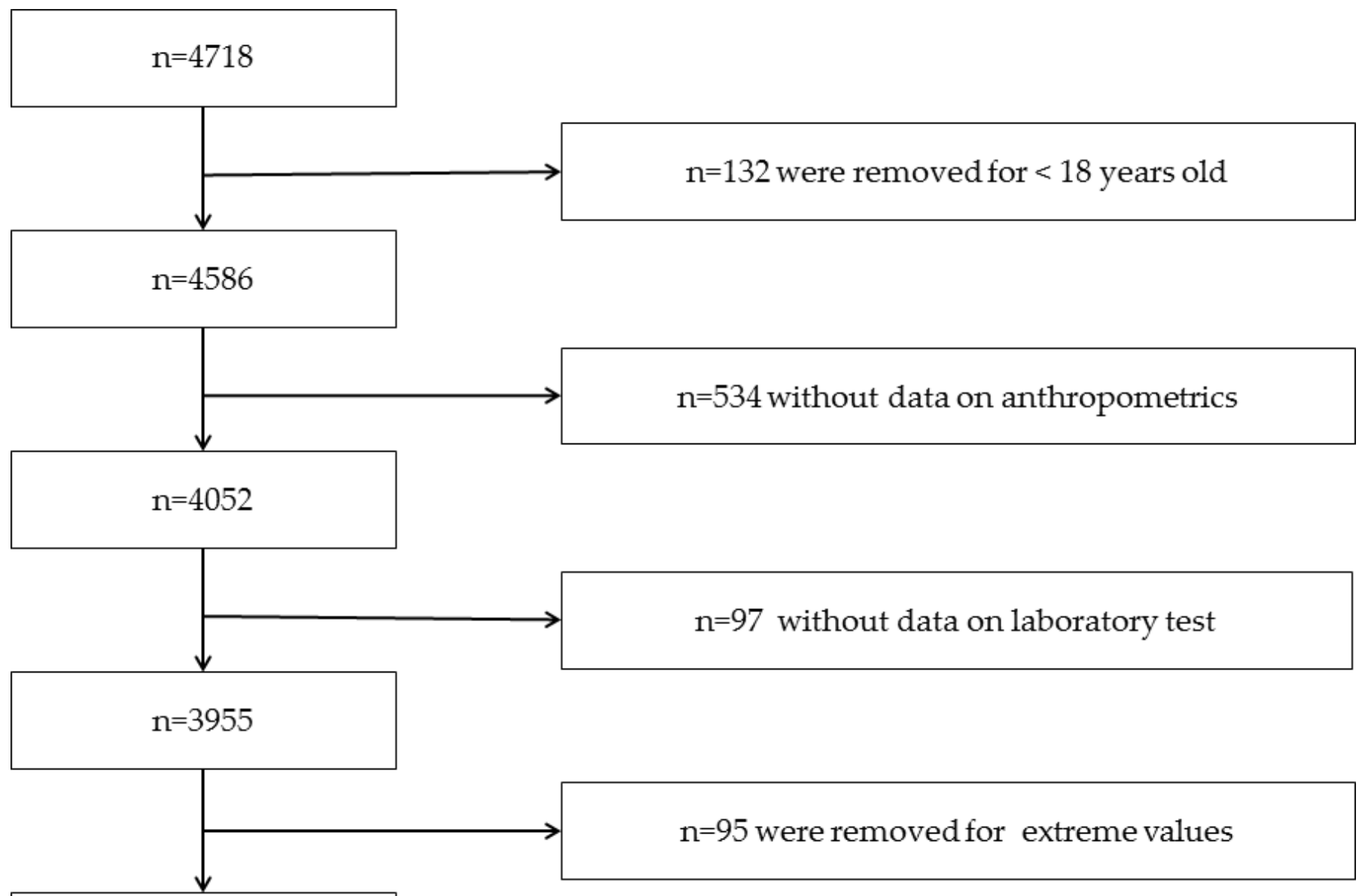

\section{$\mathrm{n}=3860$ were includedin this analysis}

Figure 1 Flow chart showing the selection of the study sample. The final sample size was 3860 adults, each with complete anthropometric, biochemical indicator and covariable data.

a questionnaire. Education levels were self-reported (no formal, primary, secondary and tertiary), marital status (married, cohabiting, other). A subject was considered a current smoker if he or she smoked regularly in the past month. A subject who consumed alcoholic drinks more than once a week was considered a current drinker. Exercise with moderate-intensity at least $150 \mathrm{~min}$ or with vigorous-intensity at least $75 \mathrm{~min}$ or an equivalent combination of moderate-intensity and vigorous-intensity activity throughout a week was defined as a regular exerciser. ${ }^{18}$ Sedentary time was positively associated with the risk of MetS. ${ }^{19}$ Therefore, we also collected the sedentary time of participants. Sedentary time was divided into four levels (Q1<2 hours, 2 hours $\leq$ Q2 $<3$ hours, 3 hours $\leq$ Q $3<5$ hours, Q4 $\geq 5$ hours) according to the quartiles.

Presence of diabetes, hypertension was defined based on clinical history, medications or, respectively, by $\mathrm{FBG} \geq 7.0 \mathrm{mmol} / \mathrm{L}$, SBP $\geq 140$ or $\mathrm{DBP} \geq 90 \mathrm{~mm} \mathrm{Hg}{ }^{20}$ Any of the following blood lipid measurements were abnormal: $\mathrm{TC} \geq 6.22 \mathrm{mmol} / \mathrm{L}$ or $\mathrm{TG} \geq 2.26 \mathrm{mmol} / \mathrm{L}$ or $\mathrm{LDL}-\mathrm{C} \geq 4.14 \mathrm{mmol} / \mathrm{L}$ or HDL-C $\leq 1.04 \mathrm{mmol} / \mathrm{L}$ or antihyperlipidemics medication use were considered as criteria for dyslipidaemia. $^{21}$

\section{Statistical analyses}

Subjects were grouped as with MetS and without MetS for men and women, separately. Continuous variables are presented as mean (SD), skewed continuous variables are shown as median (IQR) and categorical variables are described as numbers (percentages). Means, medians and proportions were compared using independent samples t tests, wilcoxon rank-sum tests and $\chi^{2}$ tests, respectively. Data normality was verified using Kolmogorov-Smirnov tests and probability plots. Violin plots were used to visualise the distribution of the anthropometric indices and its probability density ${ }^{22}$; point estimates were estimated using means and $95 \%$ CIs.

Binary logistic regression was used to evaluate the unadjusted and adjusted associations between anthropometric indices and MetS. The ORs and 95\% CIs of quartiles 2-4 (Q2-4) for each index were calculated and compared with quartile $1(\mathrm{Q} 1)$ as a reference group, unless specially 
mentioned. The receiver operator characteristic (ROC) curves were used to demonstrate the discriminatory ability of an anthropometric index over the entire range of possible values in the detection of MetS as quantified by the area under the curve (AUC). The optimal cut point for each anthropometric index in detecting MetS was chosen as the point on the curve with the highest Youden Index (sensitivity +specificity-1). The AUCs were compared using a DeLong et $a l^{23}$ non-parametric approach.

Owing to the sexual dimorphism in body composition, the analysis was performed for men and women separately. ${ }^{24}$ All models (logistic regression and ROC analysis) were adjusted for the following factors: age, alcohol consumption, sedentary time and physical activity. In addition to these factors, smoking has been added for women in the analysis. These covariables were selected in multivariable models based on clinical importance or $\mathrm{p}<0.15$ in univariate analyses. Factors not significantly associated with MetS were excluded from the final model, unless doing so resulted in OR changes of more than $10 \%$.

To test the robustness of our findings, sensitivity analyses using adjusted ROC analyses were performed: (1) owing to the strong correlation between WC and anthropometric indices, we redefined MetS: two out of four components other than WC can be diagnosed as redefined MetS; (2) stratified the age into three groups in men and women separately (age $<45,45 \leq$ age $<60$ and age $\geq 60$ ); (3) excluded the patients with dyslipidaemia, diabetes, hypertension and obesity (BMI $\left.\geq 28.0 \mathrm{~kg} / \mathrm{m}^{2}\right)$.

Statistical analyses were performed using SPSS software, V.22 (IBM) and Stata V.16.0. All statistical tests were two sided, and $\mathrm{p}<0.05$ was considered statistically significant.

\section{RESULTS}

\section{Baseline characteristics}

The characteristics of the participants, stratified by MetS in men and women, are summarised in table 1 . Of the 3860 participants, 1965 were women $(50.9 \%)$ and 1895 were men (49.1\%). MetS according to IDF was found in $41.8 \%$ of men, $42.9 \%$ of women and $42.4 \%$ of all subjects. The prevalence of MetS did not differ significantly between men and women $(p=0.508)$. The men and women with MetS tended to have significantly older age, more sedentary time, less sleep duration and higher body weight, WC, HC, when compared with the participants without MetS $(\mathrm{p}<0.001)$. SBP, DBP and FBG, TC, LDL-C, TG, non-HDL-G levels were elevated, whereas HDL-C was decreased in subjects with MetS. Likewise, MetS group had higher prevalence of dyslipidaemia, diabetes, hypertension in men and women. Among MetS components, abdominal obesity, abnormal BP and hyperglycaemic ranked in the top three in both genders. However, no statistically significant differences between the two groups were observed at smoking status and physical activity.

\section{Obesity indices according to with or without MetS}

Online supplemental figures 1 and 2 show the distribution of the anthropometric obesity indices and the mean difference for obesity indices between participants with or without MetS by gender. We found significant differences in all obesity indices in both sexes, and these subjects with MetS tended to have significantly higher anthropometric indices when compared with those participants without MetS $(\mathrm{p}<0.001)$.

Association between Mets and the level of obesity indices For the analyses of BMI, these participants classified as underweight (BMI $<18.5 \mathrm{~kg} / \mathrm{m}^{2}$ ) were excluded ( $\mathrm{n}=64$ both for males and females), and using normal weight group as a reference group. In unadjusted models, the ORs for MetS increased along with quartiles or stratifications for all ten anthropometric indices in both sexes (online supplemental figure 3). The highest unadjusted ORs for MetS in men were observed for the following indices: AVI (OR 14.65, 95\% CI 10.61 to 20.21, p $<0.001$ ), WHT.5R (OR 13.42, 95\% CI 9.73 to $18.51, \mathrm{p}<0.001$ ) and BMI $\sqrt{W C}$ (OR $12.43,95 \%$ CI 9.05 to 17.06 , p $<0.001$ ). In women, AVI had the highest unadjusted-OR for MetS (OR 16.02, 95\% CI 11.54 to 22.23, p<0.001), followed by WHtR, BRI (both OR 15.75, 95\% CI 11.35 to 21.84, $\mathrm{p}<0.001)$ and WHT.5R (OR 15.62, 95\% CI 11.22 to 21.73 , $\mathrm{p}<0.001)$.

For all analysed anthropometric indices, the ORs for MetS in adjusted models also increased in accordance with the increasing quartiles (figure 2), whereas they were slightly lower compared with those in the unadjusted models. Moreover, the risk of MetS was not significantly higher only in the second quartiles of ABSI and BAI indicator in females. In men, AVI still had the highest OR for MetS (OR 15.22, 95\% CI 10.88 to 21.30, $\mathrm{p}<0.001$ ), followed by WHT.5R (OR 13.83, 95\% CI 9.89 to $19.35, \mathrm{p}<0.001)$ and BMI $\sqrt{ } \mathrm{WC}(\mathrm{OR} 12.14,95 \%$ CI 8.79 to $16.76, \mathrm{p}<0.001)$. While, the highest ORs for the occurrence of MetS in women were noted for the following indices: BRI and WHtR (both OR 14.91, 95\% CI 10.39 to 21.4, $\mathrm{p}<0.001$ ), WHT.5R (OR $14.22,95 \%$ CI 9.96 to 20.29, $\mathrm{p}<0.001$ ) and AVI (OR 14.03, 95\% CI 9.91 to 19.87, $\mathrm{p}<0.001)$. In contrast, the lowest ORs for MetS were ABSI in both sexes. ABSI and BAI were not shown due to little linkage.

\section{ROC analysis for the obesity indices for MetS}

Table 2 shows the predictive value for each anthropometric index stratified by sex. After controlling for covariates, all adiposity indices still showed acceptable ability to identify MetS. For men, AVI tended to be more useful predictor for MetS (AUC: 0.767, sensitivity 79.6\%, specificity $69.7 \%$, cut-off point: 14.45 ), followed by WHT.5R (AUC: 0.761 , sensitivity $77.9 \%$, specificity $68.9 \%$, cut-off point: 6.54). Likewise, for women, AVI had the highest predictive value for MetS (AUC: 0.749 , sensitivity $85.4 \%$, specificity $65.2 \%$, cut-off point: 12.80 ), whereas it did not significantly differ from that of WHT.5R (AUC: 0.747, 
Table 1 Characteristics of the participants with or without MetS in men and women

\begin{tabular}{|c|c|c|c|c|c|c|}
\hline \multirow[t]{2}{*}{ Variable } & \multicolumn{3}{|l|}{ Men $(n=1895)$} & \multicolumn{3}{|l|}{ Women $(n=1965)$} \\
\hline & With MetS & Without MetS & $P$ value & With MetS & Without MetS & $P$ value \\
\hline & $793(41.8)$ & $1102(58.2)$ & - & $843(42.9)$ & $1122(57.1)$ & $0.508^{*}$ \\
\hline Age & & & $<0.001$ & & & $<0.001$ \\
\hline$<45$ & $194(24.5)$ & $383(34.8)$ & & 165 (19.6) & 448 (39.9) & \\
\hline $45-59$ & $240(30.3)$ & $299(27.1)$ & & $276(32.7)$ & $364(32.4)$ & \\
\hline$\geq 60$ & $359(45.3)$ & $420(38.1)$ & & $402(47.7)$ & $310(27.6)$ & \\
\hline Education level & & & 0.134 & & & $<0.001$ \\
\hline No formal/unknown & $120(15.1)$ & $164(14.9)$ & & $384(45.6)$ & 347 (30.9) & \\
\hline Primary & $174(21.9)$ & 227 (20.6) & & $123(14.6)$ & 195 (17.4) & \\
\hline Secondary & $373(47.0)$ & $570(51.7)$ & & $262(31.1)$ & $470(41.9)$ & \\
\hline Tertiary & $126(15.9)$ & $141(12.8)$ & & $74(8.8)$ & $110(9.8)$ & \\
\hline Married/cohabiting & 725 (91.4) & 918 (83.3) & $<0.001$ & $709(84.1)$ & $966(86.1)$ & 0.218 \\
\hline Smoking status & & & 0.699 & & & 0.216 \\
\hline Non-smoker & $580(73.1)$ & $809(73.4)$ & & $682(80.9)$ & $873(77.8)$ & \\
\hline Ex-smoker & $51(6.4)$ & $61(5.5)$ & & $6(0.7)$ & $12(1.1)$ & \\
\hline Current smoker & $162(20.4)$ & $232(21.2)$ & & $155(18.4)$ & 237 (21.1) & \\
\hline Current drinker & $154(19.4)$ & $261(23.7)$ & $<0.05$ & $43(5.1)$ & $111(9.9)$ & $<0.01$ \\
\hline Regular exerciser & $364(45.9)$ & $518(47.0)$ & 0.635 & $464(55.0)$ & $615(54.8)$ & 0.920 \\
\hline Sleep duration (h/day) & $7.07(1.33)$ & $7.18(1.43)$ & 0.087 & $7.04(1.34)$ & $7.17(1.52)$ & $<0.05$ \\
\hline Sedentary time & & & $<0.01$ & & & 0.224 \\
\hline Q1 <2 hours & $131(16.5)$ & $239(21.7)$ & & $220(26.1)$ & $282(25.1)$ & \\
\hline 2hours $\leq$ Q $<3$ hours & $144(18.2)$ & $230(20.9)$ & & $164(19.5)$ & $261(23.3)$ & \\
\hline 3 hours $\leq$ Q $3<5$ hours & 267 (33.7) & $362(32.8)$ & & $258(30.6)$ & $334(29.8)$ & \\
\hline Q4 $\geq 5$ hours & $251(31.7)$ & $271(24.6)$ & & $201(23.8)$ & $245(21.8)$ & \\
\hline \multicolumn{7}{|l|}{ Anthropometric } \\
\hline Height (m) & $1.68(0.07)$ & $1.66(0.07)$ & $<0.001$ & $1.59(7.18)$ & $1.59(6.19)$ & 0.073 \\
\hline Weight (kg) & 73.25 (10.37) & $65.15(9.94)$ & $<0.001$ & $65.15(9.88)$ & $58.26(9.02)$ & $<0.001$ \\
\hline $\mathrm{HC}(\mathrm{cm})$ & $98.52(7.03)$ & $92.80(7.26)$ & $<0.001$ & $97.59(7.29)$ & $91.48(7.41)$ & $<0.001$ \\
\hline WC (cm) & $89.56(8.19)$ & $81.18(8.46)$ & $<0.001$ & $86.50(8.53)$ & $77.57(8.84)$ & $<0.001$ \\
\hline \multicolumn{7}{|c|}{ Cardiometabolic measurements } \\
\hline $\mathrm{SBP}(\mathrm{mm} \mathrm{Hg})$ & $138.53(15.87)$ & $125.86(17.25)$ & $<0.001$ & $138.42(18.32)$ & 120.65 (17.97) & $<0.001$ \\
\hline $\mathrm{DBP}(\mathrm{mm} \mathrm{Hg})$ & $85.26(10.45)$ & $78.81(10.05)$ & $<0.001$ & 84.47 (10.59) & $75.89(9.37)$ & $<0.001$ \\
\hline FBG (mmol/L) & $5.90(5.60-6.45)$ & $5.30(4.89-5.67)$ & $<0.001$ & $5.81(5.48-6.30)$ & $5.12(4.65-5.48)$ & $<0.001$ \\
\hline $\mathrm{TC}(\mathrm{mmol} / \mathrm{L})$ & $4.15(1.14)$ & $4.02(1.00)$ & $<0.01$ & $4.42(1.20)$ & $4.21(1.08)$ & $<0.001$ \\
\hline $\mathrm{TG}(\mathrm{mmol} / \mathrm{L})$ & $1.89(1.25-2.66)$ & $1.08(0.78-1.46)$ & $<0.001$ & $1.76(1.14-2.49)$ & $1.03(0.73-1.41)$ & $<0.001$ \\
\hline LDL-C (mmol/L) & $2.43(0.81)$ & $2.46(0.78)$ & 0.308 & $2.59(0.85)$ & $2.46(0.84)$ & $<0.01$ \\
\hline $\mathrm{HDL}-\mathrm{C}(\mathrm{mmol} / \mathrm{L})$ & $1.11(0.42)$ & $1.37(0.40)$ & $<0.001$ & $1.31(0.49)$ & $1.51(0.44)$ & $<0.001$ \\
\hline Non-HDL-C (mmol/L) & $3.04(0.95)$ & $2.65(0.87)$ & $<0.001$ & $3.11(0.93)$ & $2.70(0.91)$ & $<0.001$ \\
\hline \multicolumn{7}{|l|}{ MetS components } \\
\hline Abdominal obesity & $633(79.8)$ & $336(30.5)$ & $<0.001$ & $721(85.5)$ & $396(35.3)$ & $<0.001$ \\
\hline Abnormal BP & $665(83.9)$ & $453(41.1)$ & $<0.001$ & $679(80.5)$ & $315(28.1)$ & $<0.001$ \\
\hline Hyperglycaemic & $614(77.4)$ & $306(27.8)$ & $<0.001$ & $608(72.1)$ & $202(18.0)$ & $<0.001$ \\
\hline High TG & $473(59.6)$ & $170(15.4)$ & $<0.001$ & $464(55.0)$ & $149(13.3)$ & $<0.001$ \\
\hline Low HDL-C & $380(47.9)$ & $176(16.0)$ & $<0.001$ & $486(57.7)$ & 335 (29.9) & $<0.001$ \\
\hline
\end{tabular}




\begin{tabular}{|c|c|c|c|c|c|c|}
\hline \multirow[t]{2}{*}{ Variable } & \multicolumn{3}{|c|}{ Men $(n=1895)$} & \multicolumn{3}{|c|}{ Women $(n=1965)$} \\
\hline & With MetS & Without MetS & $P$ value & With MetS & Without MetS & P value \\
\hline Dyslipidaemia & $587(74.0)$ & $321(29.1)$ & $<0.001$ & $484(57.4)$ & $273(24.3)$ & $<0.001$ \\
\hline Diabetes & $121(15.3)$ & $45(4.1)$ & $<0.001$ & $128(15.2)$ & $25(2.2)$ & $<0.001$ \\
\hline Hypertension & $447(56.4)$ & $284(25.8)$ & $<0.001$ & $446(52.9)$ & $210(18.7)$ & $<0.001$ \\
\hline
\end{tabular}

Unknown: men $(n=24)$, women $(n=24)$.

*Prevalence of men with MetS versus prevalence of women with MetS.

BP, blood pressure; DBP, diastolic blood pressure; FBG, fasting glucose; HC, hip circumference; HDL-C, high-density lipoprotein cholesterol; LDL-C, low-density lipoprotein cholesterol; MetS, metabolic syndrome; SBP, systolic blood pressure; TC, total cholesterol; TG, triglyceride; WC, waist circumference.

sensitivity $84.7 \%$, specificity $64.4 \%$, cut-off point: 6.31 ). The values of AUCs, sensitivity and specificity for WHtR, BRI and BMI $\sqrt{ }$ WC were similar, and presented acceptable ability of identifying MetS in males and females. Moreover, BMI and BMI $\sqrt{ }$ WC showed the greatest specificity in men (specificity: $74.1 \%$ and $73.6 \%$, respectively) and women (specificity: $66.1 \%$ and $67.2 \%$, respectively) for identifying MetS among all the indices we analysed. Conversely, owing to its lower specificity than other indices, the ABSI did not show better prediction, even if its sensitivity was higher than that of several indices (AUC: 0.588 , sensitivity $75.5 \%$, specificity $41.7 \%$; AUC: 0.569 , sensitivity $74.5 \%$, specificity $48.6 \%$, in men and women, respectively).

\section{Sensitivity analyses}

In sensitivity analysis (online supplemental figures 4 and 5), redefining the MetS, stratifying age or excluding the patients with dyslipidaemia, diabetes, hypertension and obesity did not affect the results significantly. For the redefined MetS, all adiposity indices showed modest ability to identify MetS, except for ABSI in men. Among them, BMI $\sqrt{W C}$ had the highest adjusted AUC in men and $\mathrm{BMI} \sqrt{\mathrm{WC}}>\mathrm{AVI}=\mathrm{BMI}(\mathrm{p}<0.05)$, whereas in women, the highest adjusted AUC were for WHT.5R $>$ AVI $>$ BMI $\sqrt{ }$ WC $(p>0.05)$. After exclusion of patients with chronic diseases and obesity, AVI had the largest adjusted AUC, followed by WHT.5R and BMI $\sqrt{W C}$, but there was no significant difference among them in both genders $(p>0.05)$. After age stratification, the largest adjusted AUCs were for AVI and WHT.5R in these groups of age $<45$ and $45 \leq$ age $<60$ in both sexes, while in the group of age $\geq 60$, the largest adjusted AUC were for AVI, BMI $\sqrt{ }$ WC and WHT.5R, and the AUCs among them were not statistically significant ( $>0.05$ ). Interestingly, the AUCs for MetS generally increased along with age stratification in men, while in women generally showed a trend of rising first and then decreasing.

\section{DISCUSSION}

This cross-sectional study comprehensively compared the association of 10 anthropometric indices with MetS in a group of Ningxia adults from northwest China. To the best of our knowledge, no report has previously investigated the association between WHT.5R, BMI $\downarrow$ WC and MetS. The main findings of this study demonstrated that WHT.5R and BMI $\sqrt{ }$ WC were reliable and easy-tocalculate anthropometric tools for estimation of obesity that were strongly related to MetS. And among the ten indices we analysed, AVI and WHT.5R show superior ability to identify MetS. The results of this study also showed the obesity indices have the different discriminatory ability by gender; therefore, it will be optimal that the sex based measurements are applied to clinical practices and public health.

In the present study, the overall prevalence of MetS was $42.4 \%$ according to IDF criteria. The prevalence was higher than Xinjiang $(21.8 \%)^{25}$ and Shaanxi $(29.4 \%),{ }^{26}$ two northwest cities in China, the national level in China $(33.9 \%)^{27}$ and Gujarati Asian Indians $(34.3 \%),{ }^{28}$ whereas, lower than elderly Colombians (58.7\%), ${ }^{29}$ similar to adults from Colombia $(41 \%) .{ }^{30}$ The possible reason may be the difference of the MetS criteria, racial or the age range of the population. The prevalence of MetS also increased with increasing age, which may be led by subtle decreases in basal metabolic rate and physical activity that are not matched with decreases in energy intake. ${ }^{31}$

We demonstrated that WHT.5R is an accurate screening tool for discriminating MetS in both sexes. This result confirms two earlier studies of Nevill et $\mathrm{al}^{1432}$, which proposed WHT.5R is a stronger predictor of abdominal adiposity and cardiometabolic risk than other anthropometric indices. Although in line with other authors, ${ }^{2933-35}$ the widely used WHtR also showed better predictive ability for MetS, we would like to emphasise it is not as precise as WHT.5R. A recent analytical review ${ }^{16}$ also confirmed the result and explained the reason. They investigated what the optimum value of $\alpha$ in the index $\mathrm{WC} /$ height $^{\alpha}$ is, proved that WHT.5R $(\alpha=0.5)$ showed minimal correlations with height and maximal correlations with $\mathrm{BF} \%$, while WHtR $(\alpha=1)$ showed the weakest associations with adiposity and the strongest associations with height among the indices they evaluated with scaling powers ranging from 0 to $1.0 .{ }^{16}$ Besides the identical variables, WC and Height, as WHT.5R and WHtR, $\varpi$ is added to calculate BRI. It should be noted that WHtR and BRI showed almost the same correlation and predictive 
Men

Women

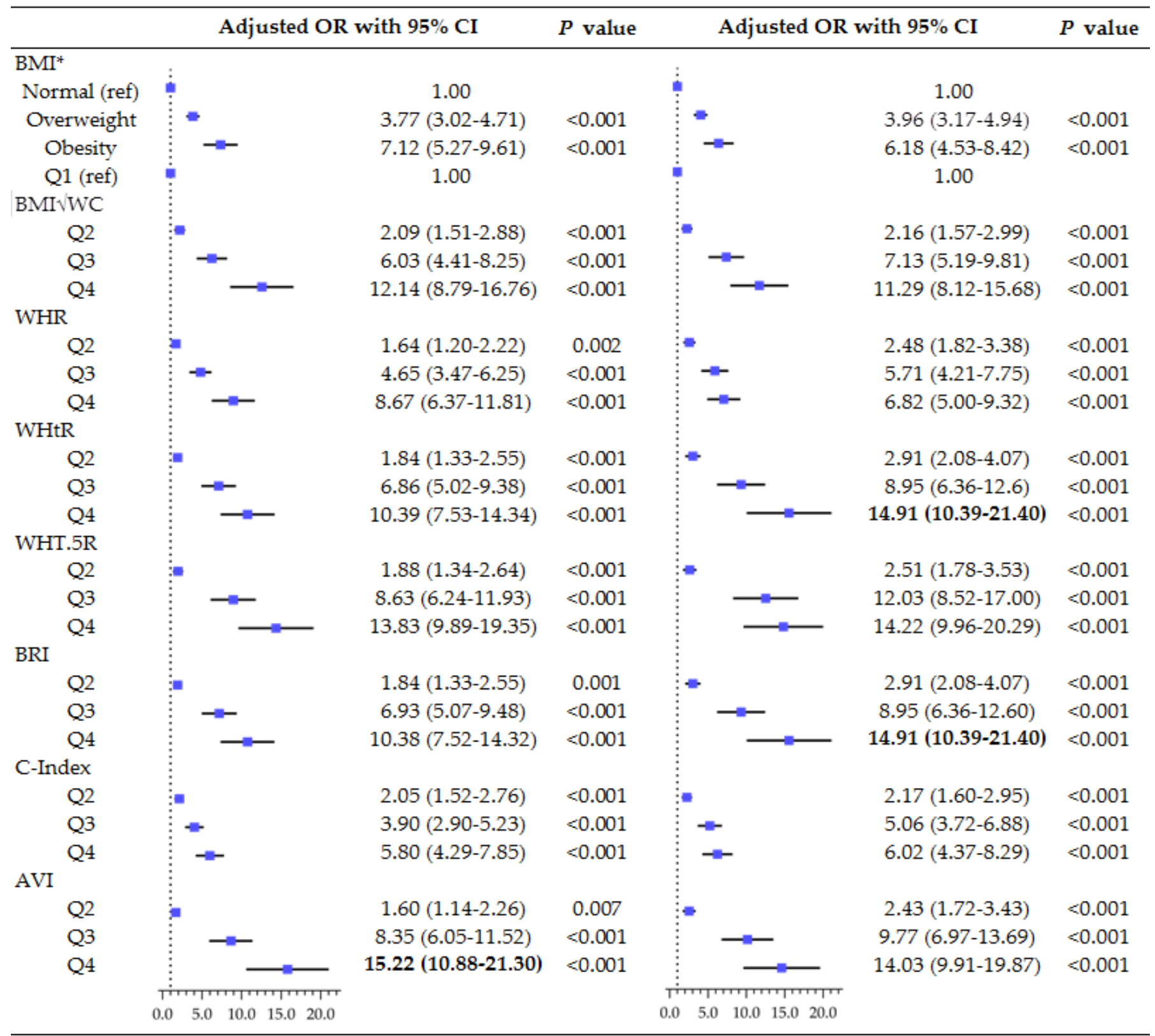

Figure 2 Adjusted ORs for MetS in quartiles of anthropometric indices in men and women. ORs adjusted for age, alcohol consumption, sedentary time and physical activity (extra added smoking status for women analysis). "Normal: $18.5 \leq \mathrm{BMl}<24 \mathrm{~kg} /$ $\mathrm{m}^{2}$; overweight: $24 \leq \mathrm{BMl}<27.9 \mathrm{~kg} / \mathrm{m}^{2}$; obesity: $\mathrm{BMI} \geq 28 \mathrm{~kg} / \mathrm{m}^{2}$. Those with a $\mathrm{BMl}<18.5 \mathrm{~kg} / \mathrm{m}^{2}$ were excluded from these analyses ( $\mathrm{n}=64$ for both men and women), and using normal weight group as a reference group. For other anthropometric indices, the first quartile (Q1) was used as a reference group. Q2, second quartile; Q3, third quartile; Q4, fourth quartile. AVI, Abdominal Volume Index; BMI, body mass index; BMI $\sqrt{ }$ WC, BMI multiply by sqrt waist circumference; BRI, Body Roundness Index; Cindex, conicity index; MetS, metabolic syndrome; WHR, waist-to-hip ratio; WHtR, waist-to-height ratio; WHT.5R, waist divided by height ${ }^{0.5}$.

ability with MetS in logistic regression and ROC analysis in both genders. This is consistent with the results that BRI and WHtR have an equal discriminating power for detecting high cardiometabolic risk in older Colombian adults reported by Ramirez-Velez $e t a l^{29}$. Taken together, a major advantage of WHT.5R over WHtR and BRI appears to be precision and simplicity. Such an advantage could translate to enhanced delivery of public health messages aimed at reducing obesity incidence, as well as the clinical and public screening of individuals at risk of metabolic abnormality.

BMI $\sqrt{ }$ WC is a novel obesity index that has just been proposed, for it could effectively predict percentage $\mathrm{BF}$ in children. ${ }^{15}$ This is the first study to apply BMI $\sqrt{ }$ WC to detect MetS in adults, and we found that there was a strong association between BMI $\sqrt{W C}$ and MetS, and it demonstrated an acceptable power for detecting MetS. Our results confirmed the study of Nevill. ${ }^{15}$ Owing to the 


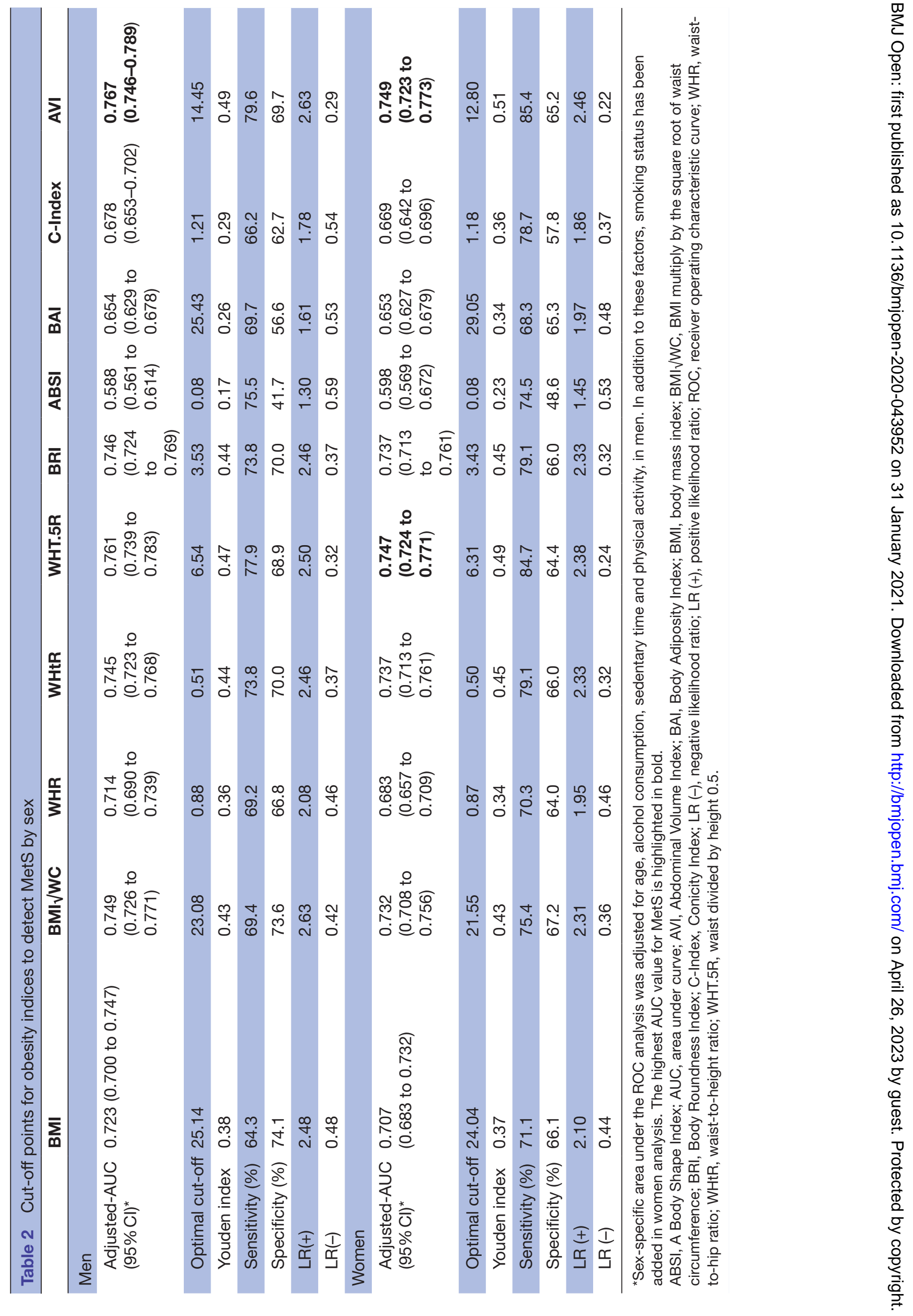


introduction of WC as a third dimension, which improves the limitations of using BMI and WC in isolation, BMI $\sqrt{ } \mathrm{WC}$ could be a more effective and equally noninvasive proxy for obesity and MetS, and the assessment of BMI $\sqrt{W C}$ is no more time-consuming or arduous than BMI and WC separately. Moreover, it is worth noting that BMI $\sqrt{ }$ WC showed the greatest specificity $(67.2 \%)$ in women and the second greatest specificity $(73.6 \%)$ in men for discriminating MetS among all the indices we analysed. Based on above discussion, it may also have some value in clinical settings and public health.

Our findings also provided strong evidence that AVI is a reliable anthropometric tool for identifying MetS in both sexes. ${ }^{36-38}$ Interestingly, in consistent with GuerreroRomero and Rodríguez-Morán ${ }^{10}$ despite the fact that the same variables, $\mathrm{WC}$ and $\mathrm{HC}$, were used to calculate the WHR and AVI, a high agreement was not obtained between the two indices from our findings. Because AVI was an estimation of overall abdominal volume that theoretically included both intra-abdominal fat and adipose tissue volume, and possessed the advantage of considering the gender-dependent fat distribution, ${ }^{10}$ it can explain the high association between AVI and MetS, as well as its superiority to WHR and other indices.

Although C-Index, BAI and ABSI showed positive associations with MetS, they are not effective screening tools for MetS in both sexs. These observations are consistent with previous studies, ${ }^{39-42}$ and a prospective, longitudinal study in Chinese adults. ${ }^{43}$ Lawrence $e t a l^{37}$ reported that C-Index showed better performance in the prediction of MetS and its components only in apparently healthy Ghanaian females. Thus, combined with our findings and that of previous studies, it can be concluded that C-Index, BAI and ABSI do not seem to be useful obesity indices for detecting MetS. Otherwise, we would like to emphasise although BRI, AVI showed a better ability to identify MetS, they are of difficult use due to the complicated formulas.

There are limitations with this study. First, this is a cross-sectional design study, with its inherent limited explanation of cause-and-effect temporality. Therefore, further cohort studies using a prospective design are needed to evaluate the relation between anthropometric measurements and MetS and the validity of the obtained cut-off values. Second, although we statistically adjusted for confounding factors, the adjustment may not have entirely removed confounding factors. Third, the effects of dyslipidaemia, hypertension, diabetes and other medications, which may be affecting the value of blood biochemical measurements, were not considered. Fourth, these participants of this study were only from a region in northwest China. Hence, these results may be not completely applicable to other populations. Finally, the present study defined MetS using IDF 2009 criteria. Therefore, further studies are needed to determine whether the results are consistent under different criteria. Despite these limitations, strengths of this paper include: (1) the large sample size, and the participants covers in each region of Ningxia, which allowed us to obtain solid results that are comparable to those of other studies; (2) the use of standardised methodological procedures in our study, which minimised measurement bias; (3) sensitivity analyses were performed and the results did not change significantly and (4) we provided gender-specific thresholds for various surrogate anthropometric indices.

\section{CONCLUSIONS}

In conclusion, results from this cross-sectional analysis indicated that WHT.5R and BMI $\sqrt{ }$ WC had significantly positive association with MetS in both sexes and AVI, WHT.5R could be useful screening tools for identifying MetS in both sexes. However, some of these indices are not easy to calculate, thus if being aided with practical software, may be potentially applied to identify at-risk individuals for further clinical evaluations. Considering the BMI and BMI $\sqrt{ }$ WC tended to higher specificity, while abdominal obesity index tended to higher sensitivity for identifying MetS, the use of both generalised and abdominal index in clinical and public health would improve the accuracy of screening obesity and health risk phenotyping beyond each index alone, which deserves further study.

Acknowledgements We would like to thank School of Public Health and Management, Ningxia Medical University. The author is also grateful for the assistance in data collection processes of the master students from School of Public Health and Management, Ningxia Medical University.

Contributors Conceptualisation: YW and $\mathrm{HL}$; methodology: YW, HL and JY; validation: YW, YF and QG; formal analysis: YW; investigation: HL, XT, YF and QG; resources; $Y F$ and $X T$; data curation: $\mathrm{HL}, \mathrm{XT}$; writing-original draft preparation, YW; writing-review and editing: YW, HL and JY; supervision: JY and XT; project administration: JY; funding acquisition: JY. All authors have read and agreed to the published version of the manuscript.

Funding This research was funded by National Natural Science Foundation of China (grant number 81060235) and Top Discipline of Public Health and Prevent Medicine (NXYLXK2017B08), Education Department of Ningxia, China.

Competing interests None declared.

Patient consent for publication Not required.

Ethics approval This study was approved by Ningixa Medical University Medical Ethnical Committee. Informed consent was obtained from all individual participants included in the study.

Provenance and peer review Not commissioned; externally peer reviewed.

Data availability statement All data relevant to the study are included in the article or uploaded as online supplemental information.

Supplemental material This content has been supplied by the author(s). It has not been vetted by BMJ Publishing Group Limited (BMJ) and may not have been peer-reviewed. Any opinions or recommendations discussed are solely those of the author(s) and are not endorsed by BMJ. BMJ disclaims all liability and responsibility arising from any reliance placed on the content. Where the content includes any translated material, BMJ does not warrant the accuracy and reliability of the translations (including but not limited to local regulations, clinical guidelines, terminology, drug names and drug dosages), and is not responsible for any error and/or omissions arising from translation and adaptation or otherwise.

Open access This is an open access article distributed in accordance with the Creative Commons Attribution Non Commercial (CC BY-NC 4.0) license, which permits others to distribute, remix, adapt, build upon this work non-commercially, and license their derivative works on different terms, provided the original work is properly cited, appropriate credit is given, any changes made indicated, and the use is non-commercial. See: http://creativecommons.org/licenses/by-nc/4.0/. 
ORCID iD

Jianjun Yang http://orcid.org/0000-0002-8332-5526

\section{REFERENCES}

1 Alberti KGMM, Eckel RH, Grundy SM, et al. Harmonizing the metabolic syndrome: a joint interim statement of the International diabetes Federation Task force on epidemiology and prevention; National heart, lung, and blood Institute; American heart association; world heart Federation; international atherosclerosis Society; and international association for the study of obesity. Circulation 2009;120:1640-5.

2 Huh JH, Yadav D, Kim JS, et al. An association of metabolic syndrome and chronic kidney disease from a 10-year prospective cohort study. Metabolism 2017;67:54-61.

3 Esposito K, Chiodini P, Colao A, et al. Metabolic syndrome and risk of cancer: a systematic review and meta-analysis. Diabetes Care 2012;35:2402-11.

4 Deedwania PC, Gupta R. Management issues in the metabolic syndrome. J Assoc Physicians India 2006;54:797-810.

5 Micklesfield LK, Goedecke JH, Punyanitya M, et al. Dual-Energy $\mathrm{X}$-ray performs as well as clinical computed tomography for the measurement of visceral fat. Obesity 2012;20:1109-14.

6 Nevill AM, Stewart AD, Olds T, et al. Relationship between adiposity and body size reveals limitations of BMI. Am J Phys Anthropol 2006;129:151-6.

7 Ashwell M, Gunn P, Gibson S. Waist-to-height ratio is a better screening tool than waist circumference and BMI for adult cardiometabolic risk factors: systematic review and meta-analysis. Obes Rev 2012;13:275-86.

8 Bergman RN, Stefanovski D, Buchanan TA, et al. A better index of body adiposity. Obesity 2011;19:1083-9.

9 Valdez R. A simple model-based index of abdominal adiposity. J Clin Epidemiol 1991;44:955-6.

10 Guerrero-Romero F, Rodríguez-Morán M. Abdominal volume index. An anthropometry-based index for estimation of obesity is strongly related to impaired glucose tolerance and type 2 diabetes mellitus. Arch Med Res 2003;34:428-32.

11 Thomas DM, Bredlau C, Bosy-Westphal A, et al. Relationships between body roundness with body fat and visceral adipose tissue emerging from a new geometrical model. Obesity 2013;21:2264-71.

12 Krakauer NY, Krakauer JC. A new body shape index predicts mortality hazard independently of body mass index. PLoS One 2012;7:e39504.

$13 \mathrm{Li} \mathrm{G}$, Wu H-K, Wu X-W, et al. The feasibility of two anthropometric indices to identify metabolic syndrome, insulin resistance and inflammatory factors in obese and overweight adults. Nutrition 2019;57:194-201.

14 Nevill AM, Duncan MJ, Lahart IM, et al. Scaling waist girth for differences in body size reveals a new improved index associated with cardiometabolic risk. Scand J Med Sci Sports 2017;27:1470-6.

15 Nevill AM, Bryant E, Wilkinson K, et al. Can waist circumference provide a new "third" dimension to BMI when predicting percentage body fat in children? Insights using allometric modelling. Pediatr Obes 2019;14:e12491.

16 Hwaung P, Heo M, Kennedy S, et al. Optimum waist circumferenceheight indices for evaluating adult adiposity: an analytic review. Obes Rev 2020;21:e12947.

17 Mach F, Baigent C, Catapano AL, et al. 2019 ESC/EAS guidelines for the management of dyslipidaemias: lipid modification to reduce cardiovascular risk. Eur Heart J 2020;41:111-88.

18 Global recommendations on physical activity for health, $2010 \mathrm{WHO}$. Available: https://apps.who.int/iris/bitstream/handle/10665/44399/ 9789241599979_eng.pdf;jsessionid=B4770A5D3464851DE3AB4234 AC2094D8? sequence=1[Accessed 14 April 2020].

19 van der Berg JD, Stehouwer CDA, Bosma H, et al. Associations of total amount and patterns of sedentary behaviour with type 2 diabetes and the metabolic syndrome: the Maastricht study. Diabetologia 2016;59:709-18.

20 Global action plan for the prevention and control of noncommunicable diseases 2013-2020, 2013WHO. Available: https://apps.who.int/iris/bitstream/handle/10665/94384/ 9789241506236_eng.pdf?sequence=1[Accessed 14 April 2020].
21 Pan L, Yang Z, Wu Y, et al. The prevalence, awareness, treatment and control of dyslipidemia among adults in China. Atherosclerosis 2016;248:2-9.

22 Rice K, Lumley T. Graphics and statistics for cardiology: comparing categorical and continuous variables. Heart 2016;102:349-55.

23 DeLong ER, DeLong DM, Clarke-Pearson DL. Comparing the areas under two or more correlated receiver operating characteristic curves: a nonparametric approach. Biometrics 1988;44:837-45.

24 Wells JCK. Sexual dimorphism of body composition. Best Pract Res Clin Endocrinol Metab 2007;21:415-30.

25 Guo S-X, Zhang X-H, Zhang J-Y, et al. Visceral adiposity and anthropometric indicators as screening tools of metabolic syndrome among low income rural adults in Xinjiang. Sci Rep 2016;6:36091.

26 Sun F, Gao B, Wang L, et al. Agreement between the JCDCG, revised NCEP-ATPIII, and IDF definitions of metabolic syndrome in a northwestern Chinese population. Diabetes Ther 2018;9:1457-68.

27 Lu J, Wang L, Li M, et al. Metabolic syndrome among adults in China: the 2010 China noncommunicable disease surveillance. J Clin Endocrinol Metab 2017;102:507-15.

28 Joshi H, Shah K, Patel P, et al. Novel indexes for diagnosing metabolic syndrome in apparently healthy Gujarati Asian Indians: a cross-sectional study. QJM 2016;109:717-22.

29 Ramírez-Vélez R, Pérez-Sousa Miguel Ángel, Izquierdo M, et al. Validation of surrogate anthropometric indices in older adults: what is the best indicator of high cardiometabolic risk factor clustering? Nutrients 2019;11:1701.

30 Davila EP, Quintero MA, Orrego ML, et al. Prevalence and risk factors for metabolic syndrome in medellin and surrounding municipalities, Colombia, 2008-2010. Prev Med 2013;56:30-4.

31 Kuk JL, Saunders TJ, Davidson LE, et al. Age-Related changes in total and regional fat distribution. Ageing Res Rev 2009;8:339-48.

32 Nevill AM, Stewart AD, Olds T, et al. A new waist-to-height ratio predicts abdominal adiposity in adults. Res Sports Med 2020;28:15-26.

33 Suliga E, Ciesla E, Głuszek-Osuch M, et al. The usefulness of anthropometric indices to identify the risk of metabolic syndrome. Nutrients 2019;11:2598.

34 Barazzoni R, Gortan Cappellari G, Semolic A, et al. Central adiposity markers, plasma lipid profile and cardiometabolic risk prediction in overweight-obese individuals. Clin Nutr 2019;38:1171-9.

35 Zhang $\mathrm{X}-\mathrm{H}$, Zhang $\mathrm{M}$, He J, et al. Comparison of anthropometric and atherogenic indices as screening tools of metabolic syndrome in the Kazakh adult population in Xinjiang. Int J Environ Res Public Health 2016;13:428.

36 Perona JS, Schmidt-RioValle J, Fernández-Aparicio Ángel, et al. Waist circumference and abdominal volume index can predict metabolic syndrome in adolescents, but only when the criteria of the International diabetes Federation are employed for the diagnosis. Nutrients 2019;11. doi:10.3390/nu11061370. [Epub ahead of print: 18 Jun 2019].

37 Quaye L, Owiredu WKBA, Amidu N, et al. Comparative abilities of body mass index, waist circumference, abdominal volume index, body adiposity index, and Conicity index as predictive screening tools for metabolic syndrome among apparently healthy Ghanaian adults. J Obes 2019;2019:1-10.

38 Perona JS, Schmidt Rio-Valle J, Ramírez-Vélez R, et al. Waist circumference and abdominal volume index are the strongest anthropometric discriminators of metabolic syndrome in Spanish adolescents. Eur J Clin Invest 2019;49:e13060.

39 Adejumo EN, Adejumo AO, Azenabor A, et al. Anthropometric parameter that best predict metabolic syndrome in South West Nigeria. Diabetes Metab Syndr 2019;13:48-54.

40 de Oliveira CC, Roriz AKC, Ramos LB, et al. Indicators of adiposity predictors of metabolic syndrome in the elderly. Ann Nutr Metab 2017;70:9-15.

41 Kuhn PC, Vieira Filho JPB, Franco L, et al. Evaluation of body adiposity index (BAl) to estimate percent body fat in an Indigenous population. Clin Nutr 2014;33:287-90.

42 Maessen MFH, Eijsvogels TMH, Verheggen RJHM, et al. Entering a new era of body indices: the feasibility of a body shape index and body roundness index to identify cardiovascular health status. PLOS One 2014;9:e107212.

43 Wang $\mathrm{H}$, Liu A, Zhao $\mathrm{T}$, et al. Comparison of anthropometric indices for predicting the risk of metabolic syndrome and its components in Chinese adults: a prospective, longitudinal study. BMJ Open 2017;7:e016062. 